\title{
Simplified Model of the Dynamic Thermal Tuning Behavior of VCSELs
}

\author{
Jia Chen, Student Member, IEEE, Andreas Hangauer, Student Member, IEEE, and \\ Markus-Christian Amann, Fellow, IEEE
}

\begin{abstract}
In this letter, a simplified thermal model is used to derive a closed-form expression for the frequency dependency of the current-to-wavelength tuning coefficient (FM response) of vertical-cavity surface-emitting lasers (VCSELs). It explains the experimentally observed square root law and shows very good agreement with measured FM responses of two different VCSEL types. It yields significantly improved accuracy compared to the usually assumed first-order low-pass model.
\end{abstract}

Index Terms-Dynamic wavelength tuning, laser theory, tunable vertical-cavity surface-emitting lasers (VCSELs).

\section{INTRODUCTION}

$\mathbf{T}$ HE DYNAMICS of the current-to-wavelength tuning coefficient of a laser diode including vertical-cavity surfaceemitting lasers (VCSELs) have usually been described with a first-order low-pass model [1]-[4]. The obtained typical values of the VCSEL thermal time constants $\tau_{t h}$ are around $1 \mu \mathrm{s}$ [4], [5], which corresponds to a 3-dB cutoff frequency of $f_{c}=$ $1 /\left(2 \pi \tau_{t h}\right) \approx 160 \mathrm{kHz}$. In a first-order low-pass model, the current-to-wavelength tuning coefficient is above cutoff frequency inversely proportional to the frequency, i.e., the tuning coefficient drops by a factor of 2 if the frequency is doubled.

Experimental characterization of the frequency dependency of the current-to-wavelength tuning coefficient, which is denoted as the frequency modulation (FM) response, has recently been carried out for VCSELs with different internal structures. It turned out that the FM response of VCSELs follows a square root law [6], i.e., above cutoff frequency the current-to-wavelength tuning coefficient drops approximately by a factor of $\sqrt{2}$ if the frequency is doubled. These characteristics have been very beneficial, e.g., for high-speed tuning at $5 \mathrm{MHz}$ of a 1680-nm VCSEL used for methane sensing [7], which would be impossible if the thermal tuning has a first-order low-pass behavior with a cutoff frequency around $160 \mathrm{kHz}$.

A theory of the FM response for distributed feedback (DFB) lasers already exists that matches the measured laser behavior

Manuscript received February 6, 2008; revised March 10, 2008. This work was supported in part by the Federal Ministry of Education and Research of Germany under Project "NOSE," Contract 13N8772, and in part by the European Union under Project "NEMIS," Contract 031845.

J. Chen and A. Hangauer are with Power and Sensor Systems, Siemens Corporate Technology, D-80200 Munich, Germany, and with the Walter Schottky Institute, Technical University of Munich, D-85748 Garching, Germany (e-mail: jia.chen@wsi.tum.de).

M.-C. Amann is with the Walter Schottky Institute, Technical University of Munich, D-85748 Garching, Germany.

Color versions of one or more of the figures in this letter are available online at http://ieeexplore.iee.org.

Digital Object Identifier 10.1109/LPT.2008.924296
[8]. For a theoretical analysis and explanation of the measured square root law of FM response of VCSELs, we developed a simplified thermal VCSEL model based on the heat equation and computed the FM response analytically. The current modulation causes an internal heating in the laser, which results in a refractive index variation in the laser material, so that the emitted wavelength changes. The active region of the VCSEL is modeled as a Gaussian-distributed instantaneous plane heat source. The average temperature in the active region over time is assumed to be proportional to the time-dependent wavelength of the laser. The FM impulse response (thermal impulse response) is therefore the variation of the average temperature in the mode volume over time, when a heat impulse is generated at time $t=0$. The Fourier transform of this impulse response then gives the FM response of the laser.

\section{Simplified Thermal VCSEL Model AND DeRIVATION OF FM RESPONSE}

For the theoretical calculations, assumptions about the heat source, conduction medium, and optical mode distribution have been made as follows.

1) Heat source: The active region generates the heat and is modeled as an infinitely thin plane heat source at $z=0$. The strength of heat generation in the infinitely thin plane is radially Gaussian distributed with the standard deviation $R_{0}$. The heat production is a $\delta$-function and occurs at $t=0$.

2) Optical mode: The optical mode is radially Gaussian distributed with the standard deviation $R_{1}$ and the average temperature of the active region affects the emitted wavelength. The $\mathrm{z}$ extension (h) of the optical field is neglected in this simplified thermal analysis, which fits well to usual VCSELs with small $h /\left(2 R_{1}\right)$ ratio.

3) Conduction medium: The medium is isotropic and extends infinitely in the $x, y$, and positive $z$ direction. The substrate acts as an ideal heat sink, i.e., it is kept at a constant temperature.

The idealized VCSEL model is shown in Fig. 1.

The equations for the transient heat distribution in the VCSEL are given by the heat equation for an isotropic solid (see [9, eq. 1.6.7 ]) with appropriate boundary condition and intensity distribution of the heat source $q$ (unit: watts per cubed meter)

$$
\begin{aligned}
& \frac{d^{2} T}{d x^{2}}+\frac{d^{2} T}{d y^{2}}+\frac{d^{2} T}{d z^{2}}+\frac{q(x, y, z)}{K} \delta(t)=\frac{1}{\kappa} \frac{d T}{d t} \\
& T(x, y, z, t)=0, \quad \text { for } z=-D \text {. }
\end{aligned}
$$

The thermal diffusivity $\kappa=K / c \rho$ is a material constant, which is a measure of transient heat flow and is defined as the thermal conductivity $K$ divided by the product of specific heat capacity 


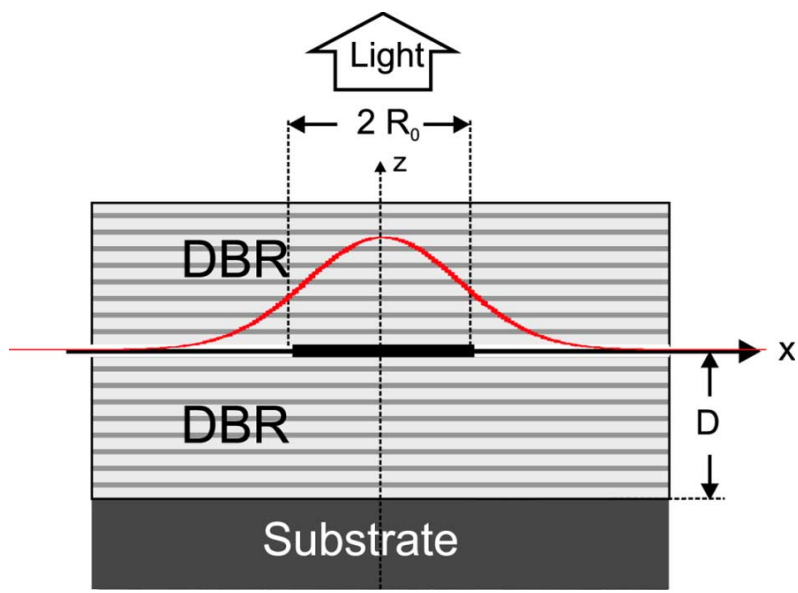

Fig. 1. Schematic cross-section in the $x-z$ plane of the idealized VCSEL structure. The active region (solid black) is modeled as an infinitely thin plane at $z=0$ that generates heat with radially Gaussian distributed intensity (standard deviation: $R_{0}$ ). The substrate is located at $z \leq-D$ and kept at a constant temperature. DBR is distributed Bragg reflector.

$c$ times density $\rho$. The plane heat source is assumed to have a Gaussian-distributed intensity, i.e.,

$$
q(x, y, z)=\delta\left(z-z_{0}\right) \frac{1}{2 \pi R_{0}^{2}} \exp \left(-\frac{x^{2}+y^{2}}{2 R_{0}^{2}}\right)
$$

whereby the source is located in the plane $z=z_{0}$ and the standard deviation of the distribution is assumed to be the current aperture radius $R_{0}$.

The transient temperature response $T_{g}(x, y, z, t)$ at an arbitrary point $(x, y, z)$ at time $t$ can be calculated by spatial convolution of the transient point heat source response and the source distribution $q(x, y, z)$ (see [9, eq. 10.2.5]). The boundary condition (2) is satisfied by using the method of images (see [9, Sec. 10.10]). With the disc heat source located at $z_{0}=0$, we associate the mirrored sink with the same strength but opposite sign at $z_{0}=-2 D$. The superposition of these two sources will give a zero temperature at $z=-D$.

The average temperature $\bar{T}_{g}(t)$ of the active region is determined by averaging $T_{g}(x, y, z, t)$ with respect to the optical mode distribution $M(x, y, z)$

$$
\bar{T}_{g}(t)=\iiint T_{g}(x, y, z, t) M(x, y, z) \mathrm{d} x \mathrm{~d} y \mathrm{~d} z .
$$

As noted in the assumptions above, the optical mode distribution is also chosen to be a Gaussian function

$$
M(x, y, z)=\delta(z) \frac{1}{2 \pi R_{1}^{2}} \exp \left(-\frac{x^{2}+y^{2}}{2 R_{1}^{2}}\right) .
$$

The FM frequency response is obtained by Fourier transforming the thermal impulse response. The Fourier transform is denoted by a hat accent.

The results are

$$
\begin{aligned}
& \bar{T}_{g}(t)=\frac{1}{4 \pi^{3 / 2} \sqrt{\kappa t}\left(R_{0}^{2}+R_{1}^{2}+2 \kappa t\right)}\left(1-\exp \left(-\frac{D^{2}}{\kappa t}\right)\right) \\
& \widehat{T}_{g}(f)=\frac{\sqrt{f_{0}}}{4 \sqrt{2} \kappa^{3 / 2}}\left(\exp \left(i \frac{f}{f_{0}}\right) \operatorname{erfc}\left(\sqrt{i \frac{f}{f_{0}}}\right)\right.
\end{aligned}
$$

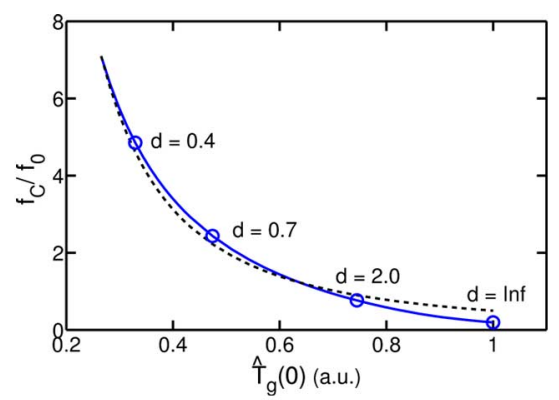

(a)

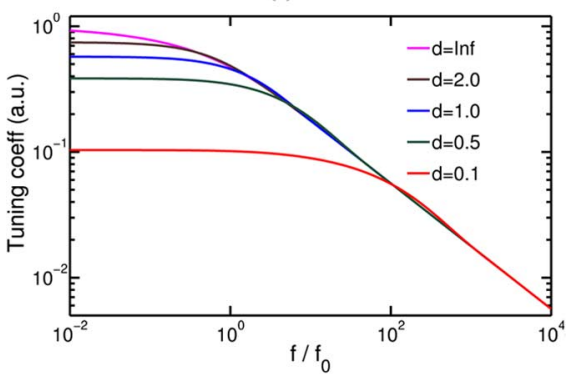

(b)

Fig. 2. (a) Exact (solid curve) and approximate [(10), broken curve] relationship of the normalized cutoff frequency $f_{c} / f_{0}$ and the tuning coefficient at dc condition with constant $R_{0}, R_{1}$, and $\kappa$, when $d$ is adjusted. (b) $\widehat{T}_{g}(f)$ for different $d$ with constant $R_{0}, R_{1}$, and $\kappa$ (curves are shown in the same order as listed in the legend).

$$
\left.-\exp \left(i \frac{f}{f_{0}}+d^{2}\right) \operatorname{erfc}\left(\sqrt{i \frac{f}{f_{0}}}+d\right)\right)
$$

and the FM response $S(f)$ is defined by

$$
S(f):=\frac{\widehat{T}_{g}(f)}{\widehat{T}_{g}(0)} .
$$

Basically, $S(f)$ depends on two parameters. First, the frequency scale parameter

$$
f_{0}:=\frac{\kappa}{\left(\pi\left(R_{0}^{2}+R_{1}^{2}\right)\right)}
$$

scales the FM response in frequency. The 3-dB cutoff frequency $f_{c}$ is approximately proportional to $f_{0}^{2}$, i.e.,

$$
f_{c} \approx \frac{f_{0}^{2}}{64 \widehat{T}_{g}(0)^{2} \kappa^{3}}
$$

or rather

$$
f_{c} \cdot \widehat{T}_{g}(0)^{2} \approx\left((8 \pi)^{2} \kappa\left(R_{0}^{2}+R_{1}^{2}\right)^{2}\right)^{-1} .
$$

If $\kappa, R_{0}$, and $R_{1}$ are held constant, the cutoff frequency $f_{c}$ has an inverse quadratic relationship with the tuning coefficient at dc condition, which is proportional to $\widehat{T}_{g}(0)$ [Fig. 2(a)].

The second parameter is the VCSEL form factor

$$
d:=\frac{D}{\sqrt{\frac{\left(R_{0}^{2}+R_{1}^{2}\right)}{2}}}
$$

which is the quotient of the distance of the active region to the substrate $D$ and the square mean of the current aperture radius 


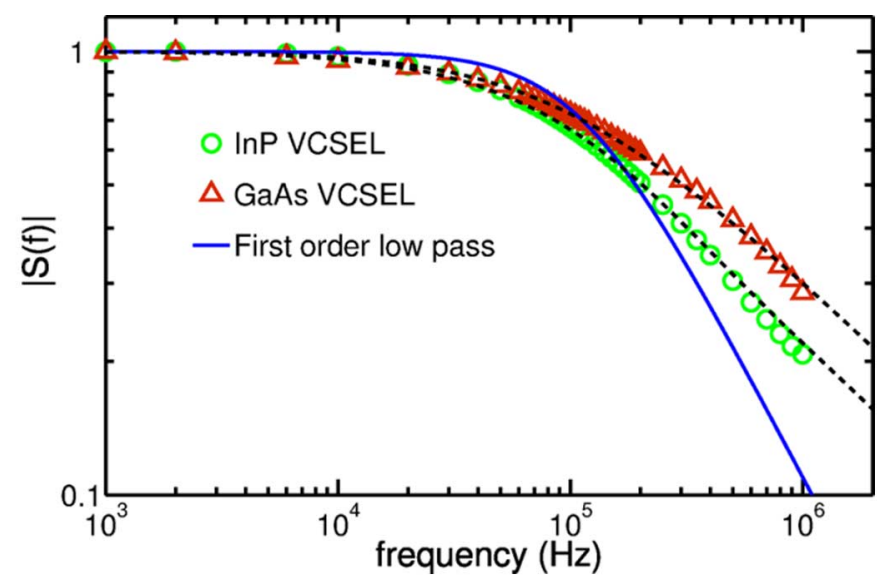

Fig. 3. Measurement data of FM response $|S(f)|$ of GaAs VCSEL (triangles) and InP VCSEL (circles) with the calculated FM response according to the simplified thermal model (broken curve). The solid curve represents the first-order low pass with the cutoff frequency $110 \mathrm{kHz}$, which is the same for the GaAs VCSEL.

$R_{0}$ and the optical mode radius $R_{1}$. A decreasing $D$ (active region nearer to the heat sink) or $d$ with constant $R_{0}, R_{1}$, and $\kappa$ increases the cutoff frequency but lowers the tuning coefficient [Fig. 2(b)]. It should be noted that the tuning behavior for high frequencies does not depend on $D$ or $d$ (constant $R$ and $\kappa$ parameters), because the curves have the same asymptote. In the simulation, the heat source and the optical mode are assumed to be both Gaussian distributed with the same radius. This is not a limitation, because according to calculations the same result for $\bar{T}_{g}(t)$ is obtained if $R_{0}^{2}+R_{1}^{2}$ is kept constant.

In the case of no boundary condition (i.e., $d \rightarrow \infty$ ), the FM response can be expressed in terms of a confluent hypergeometric function of the second kind (see [10, 13.6.39]), which is related to the generalized hypergeometric function

$$
\begin{aligned}
\left.S(f)\right|_{d \rightarrow \infty} & =\exp \left(i \frac{f}{f_{0}}\right) \operatorname{erfc}\left(\sqrt{i \frac{f}{f_{0}}}\right) \\
& =\pi^{-1 / 2} \mathrm{U}\left(\frac{1}{2}, \frac{1}{2} ; i \frac{f}{f_{0}}\right) \\
& =\pi^{-1 / 2} \frac{1}{\sqrt{\frac{i f}{f_{0}}}}{ }_{2} F_{0}\left(\frac{1}{2}, 1 ;-\frac{1}{\frac{i f}{f_{0}}}\right) .
\end{aligned}
$$

Note that $\left.\right|_{2} F_{0}(1 / 2,1 ;-1 / z) \mid$ approaches its final value 1 very quickly for $f>f_{0}$. This demonstrates that $\mathrm{U}$ has basically $1 / \sqrt{i f / f_{0}}$ behavior for $f \gg f_{0}$ and thus shows that $S(f)$ follows a square root law. For decreasing $d$ the slope of the FM response at high frequencies in doubly logarithmic scale remains at 1/2 [see Fig. 2(b)], because the second summand in (7) reaches zero very quickly for increasing $f$.

\section{COMPARISON WITH EXPERIMENTAL DATA}

In Fig. 3, measurement data of the FM response of two different types of VCSELs [6] is shown in conjunction with the theoretical model presented in this letter with fitted parameters for $f_{0}$ and $d$. The values in parentheses behind $d$ are the expected values determined by dividing the current aperture radius of the VCSEL and the approximate distance of the active region to the substrate. An expected value for $f_{0}$ [see (9)] is not known, because the average thermal conductivity as well as the average thermal diffusivity of the compound semiconductor material are difficult to obtain and beyond the scope of this letter.

1) GaAs-based VCSEL (763 nm, ULM Photonics): $R_{0}=$ $R_{1}=1.5 \mu \mathrm{m}, d=2.7$ (expected: 2.6$), f_{c}=110 \mathrm{kHz}$ $\left(f_{0}=190 \mathrm{kHz}\right)$.

2) InP-based VCSEL (1853 nm, VERTILAS): $R_{0}=R_{1}=$ $2.5 \mu \mathrm{m}, d=0.82$ (expected: 0.8$), f_{c}=85 \mathrm{kHz}\left(f_{0}=\right.$ $42 \mathrm{kHz})$.

\section{CONCLUSION}

As shown in this letter, it is possible to derive closed-form expressions for the FM impulse and frequency response that give a significantly better qualitative description of the actual device behavior than the common first-order low-pass model. The calculation is based on the assumptions of a two-dimensional Gaussian-distributed plane heat source and optical mode distribution. The analytical expression of the FM response shows a square root behavior for high frequencies and recreates the measurement result with good accuracy. Compared to the thermal properties of conventional edge emitting lasers, VCSELs show a different 3-D heat propagation, which results in this square root behavior and a much higher cutoff frequency.

\section{ACKNOWLEDGMENT}

The authors would like to thank R. Strzoda for valuable discussions on this subject.

\section{REFERENCES}

[1] R. D. Esman and D. L. Rode, "Semiconductor-laser thermal time constant," J. Appl. Phys., vol. 59, pp. 407-409, Jan. 1986.

[2] H. I. Abdelkader, H. H. Hausien, and J. D. Martin, "Temperature rise and thermal rise-time measurements of a semiconductor laser diode," Rev. Sci. Instrum., vol. 63, pp. 2004-2007, 1992.

[3] P. K. L. Chan, K. P. Pipe, Z. Mi, J. Yang, P. Bhattacharya, and D. Lüerßen, "Thermal relaxation time and heat distribution in pulsed InGaAs quantum dot lasers," Appl. Phys. Lett., vol. 89, pp. 011110-1-011110-3, Jul. 2006.

[4] P. Mena, J. Morikuni, S. Kang, A. Harton, and K. Wyatt, "A comprehensive circuit-level model of vertical-cavity surface-emitting lasers," 1999 [Online]. Available: citeseer.ist.psu.edu/mena99comprehensive. $\mathrm{html}$

[5] G. Hasnain, K. Tai, L. Yang, Y. H. Wang, R. J. Fischer, J. D. Wynn, B. Weir, N. K. Dutta, and A. Y. Cho, "Performance of gain-guided surface emitting lasers with semiconductor distributed Bragg reflectors," IEEE J. Quantum Electron., vol. 27, no. 6, pp. 1377-1385, Jun. 1991.

[6] J. Chen, A. Hangauer, R. Strzoda, and M.-C. Amann, "Experimental characterization of the frequency modulation behavior of vertical cavity surface emitting lasers," Appl. Phys. Lett., vol. 91, pp. 141105-1-141105-3, Oct. 2007.

[7] M. Lackner, G. Totschnig, F. Winter, M. Ortsiefer, M.-C. Amann, R. Shau, and J. Rosskopf, "Demonstration of methane spectroscopy using a vertical-cavity surface-emitting laser at $1.68 \mu \mathrm{m}$ with up to $5 \mathrm{MHz}$ repetition rate," Meas. Sci. Technol., vol. 14, pp. 101-106, 2003.

[8] M.-C Amann and J. Buus, Tunable Laser Diodes. Norwood, MA: Artech, 1998, pp. 94-99.

[9] H. Carslaw and J. Jaeger, Conduction of Heat in Solids. Oxford, U.K.: Clarendon, 1990

[10] M. Abramowitz and I. Stegun, Handbook of Mathematical Functions. New York: Dover, 1964. 